\title{
Analysis of the Coating surface Properties of Coated Paper
}

\author{
Ying $\mathrm{Li}^{1}$, a , Jie Zhang ${ }^{1}$, Haishan $\mathrm{Li}^{2}$, Wen juan $\mathrm{Gu}^{1}$ and Banggui $\mathrm{He}^{1}$ \\ ${ }^{1}$ Faculty of Mechanical and Electrical Engineering, Kunming University of Science and Technology, \\ Kunming, 650093, China \\ ${ }^{2}$ Kunming Richfun Printing Co., Ltd, Kunming, 650217, China \\ ${ }^{a}$ Corresponding author:haishanying@126.com
}

Keywords: Contact angle; Surface microstructure; Surface property; Surface free energy; Binder

\begin{abstract}
The surface properties of coating layer have an important influence on printing quality. In this research, the main objective was to investigate into the coating surface topography and characteristics related to binder. The surface microstructure and properties were explored in the method of numerical and visual analysis using mercury porosimetry measurment and optical contact angle measurement. The findings indicated that the binder content in the coating layer affected the pore size, depth and distribution of the coated paper surface. The conclusions were drawn that more amount of binder on the coating surface could contribute to improve the coating surface topography and the formation of even pore size and distribution, which was good to the paper surface properties and resulted in low roughness, high paper gloss, low ink absorption and high paper surface efficiency. Binder was critical to the surface topography and structure and characteristics, which played a major role in determining print gloss, print density and appearance of final printing.
\end{abstract}

\section{Introduction}

Investigations into the influence of the coating structure on ink setting rate have been widely performed in recent years [1-4]. Factors such as pore structure, coating color formulation, particle arranging and coating speed, et are important to final printing quality. Binder is the essential component of coating color recipe. The amount and type of latex binder has a significant effect on both the structure and chemical-physical properties of coated paper, which affect critical surface properties, e.g., print gloss, roughness, ink setting rate via liquid absorption, and spreading properties [5-7]. The main objective of this study was to investigate into the surface topography, structure and properties of coating layer related to binder. To this aim, the coated samples with different amount of binder in color recipes were studied, by measuring the surface free energy, surface microstructure and properties of these coatings.

\section{Experimental}

Coating. The pigment used for coating was fine Kaolin and Calcium carbonate pigments (supplied by Mao Ming Clay Company, China). The basis weight of the woodfree base paper (supplied by Dong Tang Paper Mill) was $72 \mathrm{~g} / \mathrm{m}^{2}$. The coatings of paper were composed with 50 parts kaolin pigment, 50 parts Calcium carbonate pigment and different parts carboxylic styrene-butadiene latex (supplied by BASF Company, China) with typical types of appropriate additives. The coating formulations were in Table 1. Coating was performed with a bar coater (model K303 Multi-coater, RK Print Coat Instruments Ltd, United Kingdom) with a coating speed of $4 \mathrm{~m} \mathrm{~min}^{-1}$. The coated paper was moved to a drying oven for drying with the drying temperature of $140^{\circ} \mathrm{C}$ for $1 \mathrm{~min}$.

- Coating speed $=5 \mathrm{~m} / \mathrm{min}$

- Drying temperature $=140^{\circ} \mathrm{C}$

- Drying time $=1 \mathrm{~min}$

- Coating thickness $=24 \mu \mathrm{m}$

Printing. Ink was offset printing fast-drying cyan ink (TOKA ink, Japan). Ink was transferred onto the tissue paper strips using a laboratory printing tester (model IGT Global standard Tester 2, 
America) and ink distribution apparatus (model IGT Speed Inking Unite 4) with a printing pressure of $500 \mathrm{~N}$ and a printing speed of $0.2 \mathrm{~m} / \mathrm{s}$. The amount of ink transferred onto the ink distributing roller using IGT ink injector was $0.3 \mathrm{ml}$ and the compensation was $0.075 \mathrm{ml}$ every time. The offset printing was carried out under the following conditions.

Table 1 Coating color recipes

\begin{tabular}{cccccc}
\hline Sample & $\begin{array}{c}\text { Kaolin } \\
\left(\mathrm{pph}^{*}\right)\end{array}$ & $\begin{array}{c}\text { Calcium } \\
\text { carbonate }\left(\mathrm{pph}^{*}\right)\end{array}$ & $\begin{array}{c}\text { SB } \\
\left(\mathrm{pph}^{*}\right)\end{array}$ & $\begin{array}{c}\text { Solids } \\
(\%)\end{array}$ & $\begin{array}{c}\text { Coating } \\
\text { thickness }(\mu \mathrm{m})\end{array}$ \\
\hline G1 & 50 & 50 & 20 & 60 & 24 \\
G2 & 50 & 50 & 15 & 60 & 24 \\
G3 & 50 & 50 & 10 & 60 & 24 \\
\hline
\end{tabular}

* Parts per hundred

Measurement of Contact Angle and Surface Free Energy. Contact angle measurements were carried our using apparatus equipped with a video camera and computer software(OCA 20 and SCA 20,Data Physics Instruments). Both coated surface of sampleG1, G2and G3 were investigated. Distilled water was used as probe liquid. Distilled water droplet was deposited on the paper surface by a micro-syringe. Contact angle value was measured after reaching its equilibrium on the paper surface. The surface free energy was calculated from contact angle values for coated or non-coated surface of the paper samples.

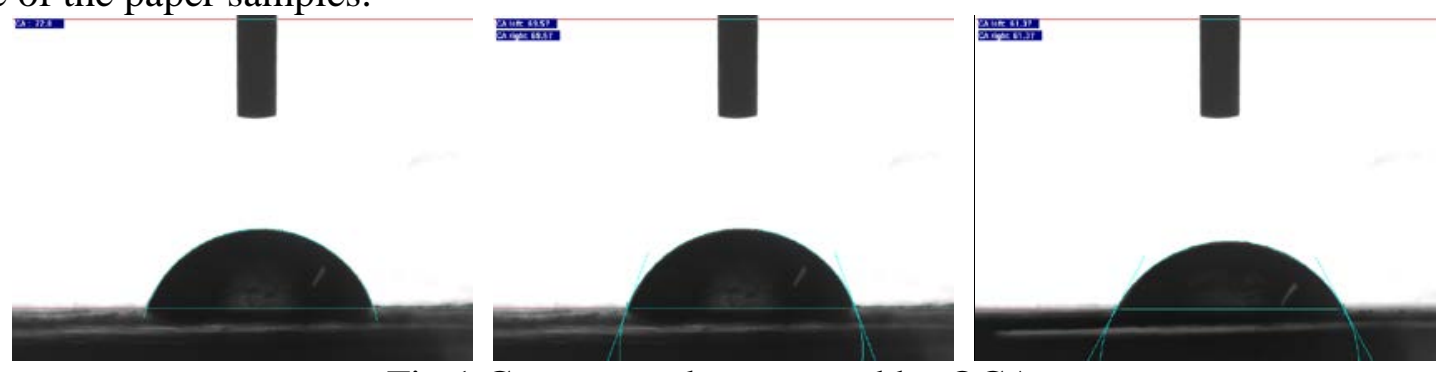

Fig.1 Contact angle measured by OCA

Mercury Porosimetry. Coating pore size distributions were determined by mercury porosimetry Measurment which was a Quantachrome Poremaster.
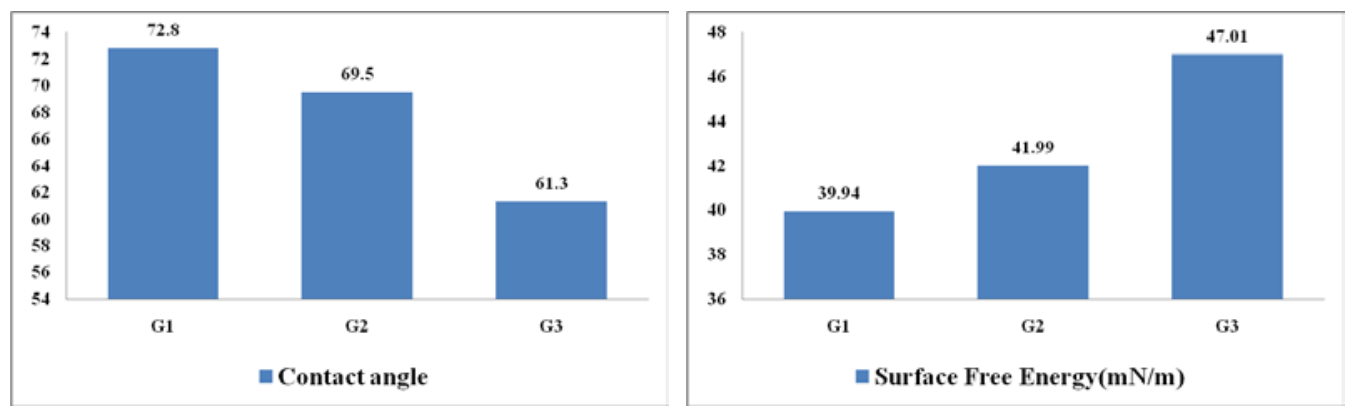

Fig.2 Contact angle and surface free energy of coated papers

\section{Results and Discussion}

Contact Angle and Surface Free Energy of Coated Paper. To characterize the surface free energy of different coating surface, contact angle measurements were used. The contact angle could be described by the Young equation [8]. The balance of the forces (surface tensions) acting the triple line Young's equation [9, 10]. Fig.1 showed images of interactions of liquid and solid in this experiment for the different coating surface. The contact angles of all the coatings were quite different shown in Fig.2. The contact angle values of sample G1, G2 and G3 with different coating surface were $72.8^{\circ}, 69.5^{\circ}$ and $61.3^{\circ}$, respectively. Surface free energy could show the surface chemical properties. Surface free energy was high and surface water hydrophilic was good, but Surface free energy was low and surface water repellency was good. Surface free energy values of sample G1, G2 and G3 were shown in Fig.2. The results indicated the surface free energy of sample G1 was lowest and surface water hydrophilic was highest due to its highest binder content. Surface free 
energy of sample G3 with 10 parts binder was higher than sample G2 with 15 parts binder, which indicated that surface water hydrophilic of sample G3 was better than sample G2.

Analyzing the Surface Microstructure. The surface pore distributions of samples were examined by mercury porosimetry measurment. It suggested form Fig. 3 that these samples differed largely in pore size distribution. Sample G1 had the lowest porosity, mean pore diameter, volume intruded and air permeability, which suggested that G1 was smoother than sample G2 and G3. Median pore diameter of sample G2 was lower than sample G3, which indicated that sample G2 had more small pores than sample G3. Porosity and Mean pore diameter of sample G2 was lower than sample G3. The conclusions were obtained that distribution and size of pore were different due to the different binder content. It result was drawn that the total pore volume, pore size, porosity and Mean pore diameter of coating surface decrease with binder content increasing. It could be concluded that the coating surfaces became smoother with the binder content adding.

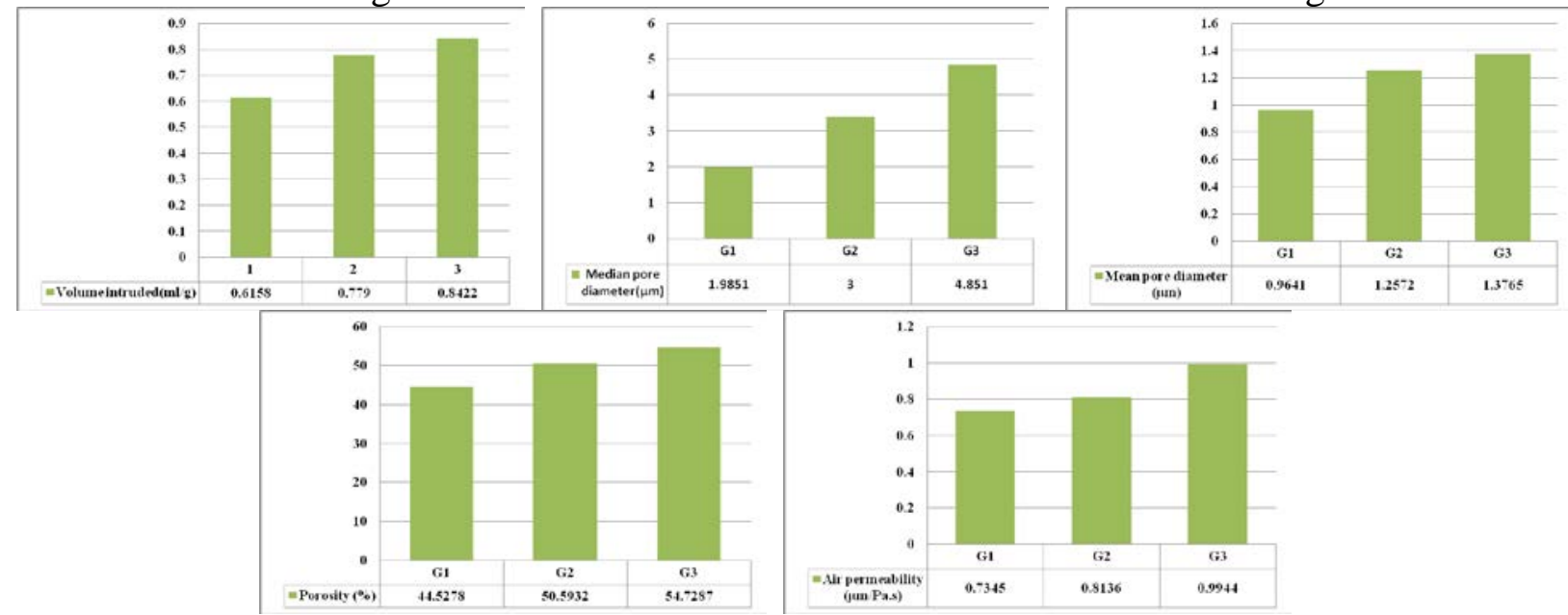

Fig.3 The porosity and pore diameter of different coatings

Coating Surface Characteristics. Changes in roughness, paper gloss, ink absorption, paper surface efficiency, and surface free energy of coating layer with the different coating layer composition were shown in Fig. 5. The results obtained were explored as the basis for discussion about the effect of binder content on surface characteristics of coating samples. Sample G1 had the lowest roughness and highest gloss compared with the other samples. The Results showed that sample G1 had the highest paper surface efficiency owing to the low ink absorption and high gloss and Sample G3 had the poorest surface properties due to different binder content. The surface free energy of sample G1 was the lowest because of its highest binder in the coating surface. The results indicated that coating surface composed with fewer binder content had more hydrophilic than coating surface composed with more binder content and more binder was benefit to improve surface properties, which made it easy for coated paper to perform printing substrate smoothly and obtain good appearance of final printing products. The more amount of binder in the consolidation phase of coating color made a contribution to the forming of the coating layer, which made the coating surface good to printing performance.
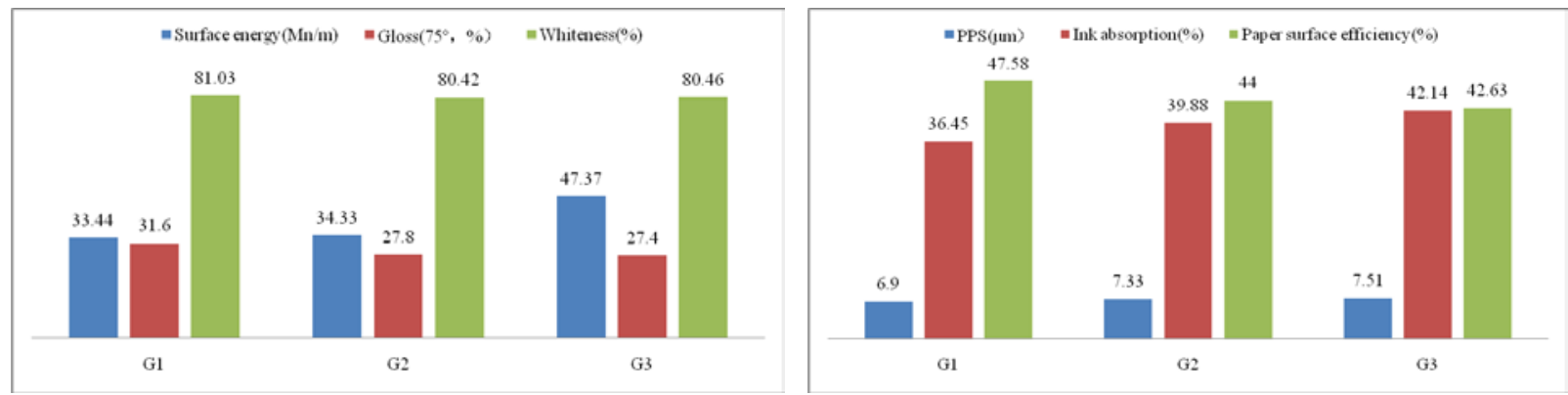

Fig.4. Coating surface printability of coated papers which include the different parts binder (G1:20 parts binder, G2:15 parts binder, G3:10 parts binder) 


\section{Conclusions}

Investigation into the relationship between surface microstructure, characteristics and binder can be obtained. The conclusions were drawn that binder affected the pore size, depth and distribution of the coated paper surface owing to the different binder accumulating condition on the coating surface. Surface water repellency increased and surface water hydrophilic decreased with amount of the binder increasing. More amount of binder in the coating was good to improve the paper properties, which resulted in low roughness, high gloss, low ink absorption, low contact angle and high paper surface efficiency. This was the reason that the coating surface with more amount of binder had high smoothness, gloss, paper surface efficiency and low ink absorption. It could be concluded that binder affected surface topography, pore distribution, surface physical-chemical properties and final printing quality of coated paper. It is obvious that what happens in the surface topography of the paper is of great important for appearance of final printing.

\section{Acknowledgements}

The authors express their gratitude to the Yunnan Province Science and Technology Department (Project KKSY 2012/01059 and KKSY 2012/01051) for financial support.

\section{References}

[1] J.S. Preston, N.J. Elton, A. Legrix, C. Nutbeem: Proceeding of TAPPI Advanced Coating Fundamentals Symposium, San Diego, May 2001.

[2] S. Rousu, P.A.C. Gane, D. Speilmann: Nordic Pulp Paper Res. J. 15 (5) (2001), p.527.

[3] J. Watanabe and P. Lepoutre: Journal of Applied Polymer Science 27 (1982) p.4207-4219.

[4] G. Engstr€om: Tappi Journal 77 (4) (1994) p.160-172.

[5] S. Di Risio and N. Yan: Collids and Surfaces A: 289(2006) p.65-74.

[6] P. Heikkil€a and N. Milosavljevic: Drying Technology 20 (1) (2002), p.211.

[7] G. Udupa, M. Singaperumal, R.S. Sirohi, M.P. Kothiyal, Meas. Sci. Technol. 11 (2000), p.315.

[8] G. Whyman, E. Bormashenko, T. SteinElton: Chem. Phys. Lett. 450 (2008) p.355.

[9] B. He, J. Lee, N. A.Patankar: Collids Surf. A. 248(2004) p.101.

[10] P. Letellier, A. Mayaffre, M. Turmine: Colloid Interface. Sci. J. 314(2007) p.604. 